\title{
The Existence of Positive Solutions for Boundary Value Problem of Nonlinear Fractional Differential Equations
}

\author{
Yanli Chen and Yongxiang $\mathrm{Li}$ \\ Department of Mathematics, Northwest Normal University, Lanzhou 730070, China \\ Correspondence should be addressed to Yongxiang Li; liyx@nwnu.edu.cn
}

Received 8 May 2014; Revised 12 August 2014; Accepted 17 August 2014; Published 27 August 2014

Academic Editor: Giovanni Anello

Copyright (c) 2014 Y. Chen and Y. Li. This is an open access article distributed under the Creative Commons Attribution License, which permits unrestricted use, distribution, and reproduction in any medium, provided the original work is properly cited.

We consider the existence of positive solutions for the nonlinear fractional differential equations boundary value problem $-D_{0^{+}}^{\alpha} u(t)=f(t, u(t)), 0<t<1, u(0)=u^{\prime}(0)=u^{\prime}(1)=0$, where $2<\alpha \leq 3$ is a real number, $D_{0^{+}}^{\alpha}$ is the Riemann-Liouville fractional derivative of order $\alpha$, and $f$ is a given continuous function. Our analysis relies on the fixed point index theory in cones.

\section{Introduction}

Fractional differential equations arise in many engineering and scientific disciplines as the mathematical modeling of systems and processes in the fields of physics, chemistry, aerodynamics, electrodynamics of complex medium, or polymer rheology; see [1-5]. The interest of the study of fractionalorder differential equations lies in the fact that fractionalorder models are more accurate than integer-order models; that is, there are more degrees of freedom in the fractionalorder models. Recently, there are some papers dealing with the existence of solutions (or positive solution) of nonlinear initial value problems of fractional differential equations by the use of techniques of nonlinear analysis (fixed-point theorems, Leray-Schauder theory, lower and upper solution method, Adomian decomposition method, ect.); see [6-15].

The famous viscous liquid flow problems in the fields of integer-order differential equations can be described by thirdorder ordinary differential equation boundary value problem

$$
\begin{gathered}
-u^{\prime \prime \prime}(t)=f(t, u(t)), \quad 0<t<1, \\
u(0)=u^{\prime}(0)=u^{\prime}(1)=0,
\end{gathered}
$$

where $f:[0,1] \times[0,+\infty) \rightarrow[0,+\infty)$ is continuous $[16-18]$. However, there are only a few exisitng contributions, as far as we know, in the field of fractional-order differential equation. In this paper, we discuss the existence of positive solution for the nonlinear fractional differential equations boundary value problem (BVP)

$$
\begin{aligned}
-D_{0^{+}}^{\alpha} u(t) & =f(t, u(t)), \quad 0<t<1, \\
u(0) & =u^{\prime}(0)=u^{\prime}(1)=0,
\end{aligned}
$$

where $2<\alpha \leq 3$ is a real number, $D_{0^{+}}^{\alpha}$ is the RiemannLiouville fractional derivative, and $f:[0,1] \times[0, \infty) \rightarrow$ $[0, \infty)$ is a continuous function.

For a more general case, specially,

$$
\begin{gathered}
D_{0^{+}}^{\alpha} u(t)+\lambda a(t) f(u(t))=0, \\
0<t<1, \quad 2<\alpha \leq 3, \\
u(0)=u^{\prime}(0)=u^{\prime}(1)=0,
\end{gathered}
$$

where $a:(0,1) \rightarrow[0,+\infty)$ is continuous with $\int_{0}^{1} a(t) d t>0$, $f \in C([0,+\infty),[0,+\infty))$ and $D_{0^{+}}^{\alpha}$ is the Riemann-Liouville fractional derivative; El-Shahed [20] obtained the existence and nonexistence of positive solutions by employing the well-known Guo-Krasnoselskii fixed point theorem of cone extension or compression. The purpose of this paper is to extend this result. Our argument is based on the fixed point index theory, which is more precise than the fixed point theorem of cone extension or compression. We will employ the theory of fixed point index in cones to present some more extensive conditions on $f$ guaranteeing the existence 
of positive solution of the BVP (2). As far as we know, the method of the fixed point index theory is firstly applied to BVP (2).

This paper is organized as follows. In Section 2, we introduce some basic definitions and properties, preliminary results that will be used to prove our main results. In Section 3, we obtain the existence of the positive solutions for BVP (2) by using the fixed point index theory.

\section{Preliminaries}

In this section, we introduce some preliminary facts which are used throughout this paper. For details, see [19].

Definition 1 (see [19]). The Riemann-Liouville fractional derivative of order $\alpha>0$ of a continuous function $f:(0,+\infty) \rightarrow$ $\mathbb{R}$ is given by

$$
D_{0^{+}}^{\alpha} f(t)=\frac{1}{\Gamma(n-\alpha)} \frac{d^{n}}{d t^{n}} \int_{0}^{t} \frac{f(s)}{(t-s)^{\alpha-n+1}} d s,
$$

where $\Gamma(\cdot)$ is Gamma function and $n=[\alpha]+1,[\alpha]$ denotes the integer part of number $\alpha$, provided that the right side is pointwise defined on $(0,+\infty)$.

Definition 2 (see [19]). The Riemann-Liouville fractional integral of order $\alpha>0$ of a function $f:(0,+\infty) \rightarrow \mathbb{R}$ is given by

$$
I_{0^{+}}^{\alpha} f(t)=\frac{1}{\Gamma(\alpha)} \int_{0}^{t}(t-s)^{\alpha-1} f(s) d s,
$$

provided that the right side is pointwise defined on $(0,+\infty)$.

Lemma 3. Let $0<\alpha<1$, if $u \in C(0,1) \cap L^{1}(0,1)$; then the fractional differential equation

$$
D_{0^{+}}^{\alpha} u(t)=0
$$

has unique solutions $u(t)=c_{1} t^{\alpha-1}+c_{2} t^{\alpha-2}+\cdots+c_{n} t^{\alpha-n}, c_{i} \in \mathbb{R}$, $i=1,2, \ldots, n$, where $n$ is the smallest integer greater than or equal to $\alpha$.

Lemma 4. Assume that $u \in C(0,1) \cap L^{1}(0,1)$ with a fractional derivative of order $\alpha>0$ that belongs to $C(0,1) \cap L^{1}(0,1)$. Then

$$
I_{0^{+}}^{\alpha} D_{0^{+}}^{\alpha} u(t)=u(t)+c_{1} t^{\alpha-1}+c_{2} t^{\alpha-2}+\cdots+c_{n} t^{\alpha-n},
$$

for some $c_{i} \in \mathbb{R}, i=1,2, \ldots, n$, where $n$ is the smallest integer greater than or equal to $\alpha$.

In the following, we present the Green's function of fractional differential equation boundary value problem.

Lemma 5 (see [20]). Let $y \in C[0,1]$ and $2<\alpha \leq 3$. The linear fractional differential equation boundary value problem

$$
\begin{gathered}
-D_{0^{+}}^{\alpha} u(t)=y(t), \quad 0<t<1, \\
u(0)=u^{\prime}(0)=u^{\prime}(1)=0
\end{gathered}
$$

has a unique solution

$$
u(t)=\int_{0}^{1} G(t, s) y(s) d s
$$

where

$$
G(t, s)= \begin{cases}\frac{t^{\alpha-1}(1-s)^{\alpha-2}-(t-s)^{\alpha-1}}{\Gamma(\alpha)}, & 0 \leq s \leq t \leq 1, \\ \frac{t^{\alpha-1}(1-s)^{\alpha-2}}{\Gamma(\alpha)}, & 0 \leq t \leq s \leq 1 .\end{cases}
$$

Lemma 6 (see [20]). Let $G$ be Green's function related to problem (8), which is given by the expression (10). Then, for all $\alpha \in(2,3]$, the following properties are fulfilled:

(1) $G(t, s)>0, \forall t, s \in(0,1)$;

(2) $G(1, s) \geq G(t, s), \forall t, s \in[0,1]$;

(3) $G(t, s) \geq t^{\alpha-1} G(1, s), \forall t, s \in[0,1]$;

(4) $G(t, s)$ is a continuous function, $\forall t, s \in[0,1]$.

Let $E=C[0,1]$ be the Banach space endowed with the norm $\|u\|=\max _{0 \leq t \leq 1}|u(t)|$. We define the operator $A$ : $C[0,1] \rightarrow C[0,1]$ by

$$
A u(t):=\int_{0}^{1} G(t, s) f(s, u(s)) d s,
$$

where $G$ is the Green's function defined in (10).

It is clear, form Lemma 5, that the nontrivial fixed points of operator A coincide with the positive solutions of BVP (2).

Let $C^{+}[0,1]=\{u \in C[0,1] \mid u(t) \geq 0, t \in[0,1]\}$. Define a cone $P \subset E$ by

$$
P=\left\{u \in C^{+}[0,1] \mid u(t) \geq t^{\alpha-1}\|u\|, \forall t \in[0,1]\right\} .
$$

Lemma 7. $A: P \rightarrow P$ is completely continuous.

Proof. From the continuity and the nonnegativeness of functions $G$ and $f$ on their domains of definition, we have that if $u \in P$, then $A u \in E$ and $A u(t) \geq 0$ for all $t \in[0,1]$; from properties (2) and (3) of Lemma 6, for all $u \in P$,

$$
\begin{aligned}
(A u)(t) & =\int_{0}^{1} G(t, s) f(s, u(s)) d s \\
& \geq \int_{0}^{1} t^{\alpha-1} G(1, s) f(s, u(s)) d s \\
& \geq t^{\alpha-1} \max _{0 \leq t \leq 1} \int_{0}^{1} G(t, s) f(s, u(s)) d s \\
& =t^{\alpha-1}\|A u\|, \quad \forall t, s \in[0,1] .
\end{aligned}
$$

Hence, $A(P) \subset P$.

Next, we show that $A$ is uniformly bounded.

Let $\Omega \subset P$ be bounded, which is to say, there exists a positive constant $M>0$ such that $\|u\|_{\infty} \leq M$ for all $u \in \Omega$. Define now

$$
L=\max _{0 \leq t \leq 1,0 \leq u \leq M}|f(t, u)|+1
$$


Then, for all $u \in \Omega$, it is satisfied that

$$
\begin{aligned}
|A u(t)| & \leq \int_{0}^{1} G(t, s) f(s, u(s)) d s \\
& \leq L \int_{0}^{1} G(t, s) d s, \quad \forall t \in[0,1] .
\end{aligned}
$$

That is, the set $A(\Omega)$ is bounded in $E$.

Finally, we show that $A$ is equicontinuous.

For each $u \in \Omega$, we have

$$
\begin{aligned}
\left|(A u)^{\prime}(t)\right|= & -\int_{0}^{t} \frac{(\alpha-1)(t-s)^{\alpha-2}}{\Gamma(\alpha)} f(s, u(s)) d s \\
& +\int_{0}^{1} \frac{(\alpha-1)[t(1-s)]^{\alpha-2}}{\Gamma(\alpha)} f(s, u(s)) d s \mid \\
\leq & \frac{\alpha-1}{\Gamma(\alpha)} \int_{0}^{t}(t-s)^{\alpha-2}|f(s, u(s))| d s \\
& +\frac{\alpha-1}{\Gamma(\alpha)} \int_{0}^{1}[t(1-s)]^{\alpha-2}|f(s, u(s))| d s \\
\leq & \frac{(\alpha-1) L}{\Gamma(\alpha)} \int_{0}^{t}(t-s)^{\alpha-2} d s \\
& +\frac{(\alpha-1) L}{\Gamma(\alpha)} \int_{0}^{1}[t(1-s)]^{\alpha-2} d s \\
\leq & \frac{L}{\Gamma(\alpha)}+\frac{L}{\Gamma(\alpha)}=\frac{2 L}{\Gamma(\alpha)}:=\bar{M} .
\end{aligned}
$$

As consequence, for all $t_{1}, t_{2} \in[0,1], t_{1}<t_{2}$, we have

$$
\left|(A u)\left(t_{2}\right)-(A u)\left(t_{1}\right)\right| \leq \int_{t_{1}}^{t_{2}}\left|(A u)^{\prime}(s)\right| d s \leq \bar{M}\left(t_{2}-t_{1}\right)
$$

Hence the set $A(\Omega)$ is equicontinuous.

Now, from the Arzela-Ascoli Theorem, we conclude that $A(\Omega)$ is relatively compact. Hence, $A: P \rightarrow P$ is a completely continuous operator.

Define an operator $T: C[0,1] \rightarrow C[0,1]$ by

$$
T u(t):=\int_{0}^{1} G(t, s) u(s) d s .
$$

Clearly, $T$ also is a completely continuous linear operator and $T(P) \subset P$.

Lemma 8. The operator $T: C[0,1] \rightarrow C[0,1]$ defined by (18) satisfies

$$
\|T\| \leq \frac{1}{(\alpha-1) \Gamma(\alpha+1)} .
$$

Proof. Let $u \in C[0,1]$. For every $t \in[0,1]$, by the definition of $T$,

$$
\begin{aligned}
|T u(t)| & =\left|\int_{0}^{1} G(t, s) u(s) d s\right| \\
& \leq \int_{0}^{1} G(t, s)|u(s)| d s \\
& \leq \int_{0}^{1} G(t, s) d s\|u\| \\
& =\frac{1}{\Gamma(\alpha)}\left[\int_{0}^{t}\left(t^{\alpha-1}(1-s)^{\alpha-2}-(t-s)^{\alpha-1}\right) d s\right. \\
& =\frac{1}{\Gamma(\alpha)}\left(\frac{1}{\alpha-1} t^{\alpha-1}-\frac{1}{\alpha} t^{\alpha}\right)\|u\| \\
& <\frac{1}{\Gamma(\alpha)} \cdot \frac{1}{\alpha(\alpha-1)}\|u\| \\
& =\frac{1}{(\alpha-1) \Gamma(\alpha+1)}\|u\| .
\end{aligned}
$$

Hence,

$$
\|T u\| \leq \frac{1}{(\alpha-1) \Gamma(\alpha+1)}\|u\| .
$$

This implies that

$$
\|T\| \leq \frac{1}{(\alpha-1) \Gamma(\alpha+1)} .
$$

The proof is completed.

Hereafter, we use $r(T)$ to denote the spectral radius of the operator $T$.

Lemma 9. Suppose that $T$ is defined by (18); then the spectral radius $r(T)>0$.

Proof. Set $m=\min _{1 / 4 \leq t, s \leq 3 / 4} G(t, s)$. Then, by (18) and the positivity of $G(t, s)$, we have $m>0$ and

$$
\begin{aligned}
T(1)(t) & =\int_{0}^{1} G(t, s) d s \\
& \geq \int_{1 / 4}^{3 / 4} G(t, s) d s \geq \frac{1}{2} m, \quad t \in\left[\frac{1}{4}, \frac{3}{4}\right], \\
T^{2}(1)(t) & =\int_{0}^{1} G(t, s) T(1)(s) d s \\
& \geq \frac{1}{2} m \int_{0}^{1} G(t, s) d s \geq\left(\frac{1}{2} m\right)^{2}, \quad t \in\left[\frac{1}{4}, \frac{3}{4}\right],
\end{aligned}
$$


Inductively, we obtain that

$$
T^{n}(1)(t) \geq\left(\frac{1}{2} m\right)^{n}, \quad t \in\left[\frac{1}{4}, \frac{3}{4}\right], n=1,2, \ldots
$$

Consequently,

$$
\left\|T^{n}(1)\right\| \geq T^{n}(1)\left(\frac{1}{2}\right) \geq\left(\frac{1}{2} m\right)^{n}, \quad n=1,2, \ldots .
$$

So,

$$
\left\|T^{n}\right\| \geq\left\|T^{n}(1)\right\| \geq\left(\frac{1}{2} m\right)^{n}, \quad n=1,2, \ldots .
$$

By this and Gelfand's formula of spectral radius we have

$$
r(T)=\lim _{n \rightarrow \infty}\left\|T^{n}\right\|^{1 / n} \geq\left(\frac{1}{2} m\right)>0 .
$$

The proof of Lemma 9 is completed.

Now, since the operator $T: C[0,1] \rightarrow C[0,1]$ is a completely continuous linear operator, by the well-known Krein-Rutman theorem ([21], Theorem 19.3), the operator $T$ has the maximum positive real eigenvalue $r(T)$; then there exists a eigenfunction $\varphi_{1} \in P \backslash\{\theta\}$ such that $T \varphi_{1}=r(T) \varphi_{1}$.

Set $\lambda_{1}=(r(T))^{-1}$; then $\varphi_{1}=\lambda_{1} T \varphi_{1}$. Thus, $\lambda_{1}$ is the minimum positive real eigenvalue of the linear equation (8).

To prove the existence of at least one positive solution of BVP (2), we will find the nonzero fixed point of $A$ (defined in (11)) by using the fixed point index theory in cones.

We recall some concepts and conclusions on the fixed point index in cones in $[21,22]$, which will be used in the argument later. Let $E$ be a Banach space and let $P \subset E$ be a closed convex cone in $E$. Assume that $\Omega$ is a bounded open subset of $E$ with boundary $\partial \Omega$ and $P \cap \Omega \neq \emptyset$. Let $A: P \cap \bar{\Omega} \rightarrow$ $P$ be a completely continuous mapping. If $A u \neq u$ for every $u \in P \cap \partial \Omega$, then the fixed point index $i(A, P \cap \Omega, P)$ is well defined. One important fact is that if $i(A, P \cap \Omega, P) \neq 0$, then $A$ has a fixed point in $P \cap \Omega$.

For $r>0$, let $P_{r}=\{u \in P \mid\|u\|<r\}$, and $\partial P_{r}=\{u \in$ $P \mid\|u\|=r\}$, which is the relative boundary of $P_{r}$ in $P$. The following two lemmas are needed in our argument.

Lemma 10 (see [22]). Let $A: P \rightarrow P$ be a completely continuous mapping. If there exists an $u_{0} \in P \backslash\{\theta\}$ such that

$$
u-A u \neq \mu u_{0}, \quad \forall u \in \partial P_{r}, \mu \geq 0,
$$

then the fixed point index $i\left(A, P_{r}, P\right)=0$.

Lemma 11 (see [22]). Let $A: P \rightarrow P$ be a completely continuous mapping. If

$$
A u \neq \mu u, \quad \forall u \in \partial P_{r}, \mu \geq 1,
$$

then the fixed point index $i\left(A, P_{r}, P\right)=1$.

Lemma 12 (see [22]). Let $A: P \rightarrow P$ be a completely continuous mapping and it satisfies that $A u \neq u$ for every $u \in \partial P_{r}$. If $\|A u\| \geq\|u\|, \forall u \in \partial P_{r}$, then the fixed point index $i\left(A, P_{r}, P\right)=$ 0 .

\section{Main Results}

In this section we show the existence of positive solutions of BVP (2) by using the fixed point index theory in cones.

Theorem 13. Assume $f:[0,1] \times[0, \infty) \rightarrow[0, \infty)$ is continuous and satisfies the following conditions.

(F1) There exist $b>\lambda_{1}$ and $\delta>0$, such that

$$
f(t, x) \geq b x, \quad \forall t \in[0,1], 0 \leq x \leq \delta .
$$

(F2) There exist $0<a<\lambda_{1}$ and $h \in C^{+}[0,1]$, such that

$$
f(t, x) \leq a x+h(t), \quad \forall t \in[0,1], x \geq 0 .
$$

Then, the BVP (2) has at least one positive solution.

Proof. Let $\varphi_{1}$ be the positive eigenfunction of $T$ corresponding to $\lambda_{1}$; thus $\varphi_{1}=\lambda_{1} T \varphi_{1}$, where $T$ is defined by (18).

Choose $r \in(0, \delta)$, where $\delta$ is the constant in assumption (F1). For every $u \in \partial P_{r}$, from assumption (F1), we have

$$
\begin{aligned}
(A u)(t) & =\int_{0}^{1} G(t, s) f(s, u(s)) d s \\
& \geq b \int_{0}^{1} G(t, s) u(s) d s \\
& >\lambda_{1} \int_{0}^{1} G(t, s) u(s) d s \\
& =\lambda_{1}(T u)(t), \quad t \in[0,1] .
\end{aligned}
$$

Namely,

$$
A u \geq \lambda_{1} T u, \quad \forall t \in[0,1], \forall u \in \partial P_{r}
$$

Suppose that $A$ has no fixed point on $\partial P_{r}$ (otherwise, the proof is completed). Now we show that

$$
u-A u \neq \mu \varphi_{1}, \quad \forall u \in \partial P_{r}, \mu \geq 0 .
$$

If it is not true, there exist $u_{0} \in \partial P_{r}$ and $\mu_{0}>0$ (if $\mu_{0}=0$, the proof is completed) such that

$$
u_{0}-A u_{0}=\mu_{0} \varphi_{1}
$$

Then,

$$
u_{0}=A u_{0}+\mu_{0} \varphi_{1}>\mu_{0} \varphi_{1}
$$

That is,

$$
u_{0}(t) \geq \mu_{0} \varphi_{1}(t) \text {. }
$$

Let

$$
\mu^{*}=\sup \left\{\mu \mid u_{0} \geq \mu \varphi_{1}, \mu>0\right\} .
$$

It is easy to see that $0<\mu_{0} \leq \mu^{*}<+\infty$ and $u_{0} \geq \mu^{*} \varphi_{1}$. Taking into account the positivity of the Green's function $G(t, s)$ and 
definition of the operator $T$, it is easy to know that $T$ is a nondecreasing linear operator, so

$$
\lambda_{1} T u_{0} \geq \mu^{*} \lambda_{1} T \varphi_{1}=\mu^{*} \varphi_{1} .
$$

Therefore by (33)

$$
u_{0}=A u_{0}+\mu_{0} \varphi_{1} \geq \lambda_{1} T u_{0}+\mu_{0} \varphi_{1} \geq\left(\mu^{*}+\mu_{0}\right) \varphi_{1},
$$

which contradicts the definition of $\mu^{*}$. Hence (34) holds and we have from Lemma 10 that

$$
i\left(A, P_{r}, P\right)=0 \text {. }
$$

On the other hand, we choose $R>r>0$. Now we show that if $R$ is large enough, then

$$
u \neq \mu A u, \quad \forall u \in \partial P_{R}, \quad 0<\mu \leq 1 .
$$

From (F2), $a<\lambda_{1}$; then there exist $0<\sigma<1$, such that $a=\sigma \lambda_{1}$.

Let $T_{2} u:=\sigma \lambda_{1} T u, u \in C[0,1]$. Then $T_{2}: C[0,1] \rightarrow$ $C[0,1]$ is a bounded linear operator and $T_{2}(P) \subset P$.

Let

$$
W=\{u \in P \mid u=\mu A u, 0<\mu \leq 1\} .
$$

In the following, we prove that $W$ is bounded.

For any $u \in W, t \in[0,1]$, we have

$$
\begin{aligned}
u(t) & =\mu(A u)(t) \\
& =\mu \int_{0}^{1} G(t, s) f(s, u(s)) d s \\
& \leq a \int_{0}^{1} G(t, s) u(s) d s+\int_{0}^{1} G(t, s) h(s) d s \\
& =\sigma \lambda_{1} \int_{0}^{1} G(t, s) u(s) d s+\int_{0}^{1} G(t, s) h(s) d s \\
& \leq\left(T_{2} u\right)(t)+\frac{\|h\|}{(\alpha-1) \Gamma(\alpha+1)} .
\end{aligned}
$$

Thus,

$$
\left(\left(I-T_{2}\right) u\right)(t) \leq \frac{\|h\|}{(\alpha-1) \Gamma(\alpha+1)}, \quad t \in[0,1] .
$$

Since $0<\sigma<1$, therefore $\sigma \lambda_{1}<\lambda_{1}$; it is easy to get that the inverse operator $\left(I-T_{2}\right)^{-1}$ exists and

$$
\left(I-T_{2}\right)^{-1}=I+T_{2}+T_{2}^{2}+T_{2}^{3}+\cdots+T_{2}^{n}+\cdots .
$$

It follows from $T_{2}(P) \subset P$ that $\left(I-T_{2}\right)^{-1} \subset P$. Hence we have

$$
\|u\| \leq\left\|\left(I-T_{2}\right)^{-1}(1)\right\| \cdot \frac{\|h\|}{(\alpha-1) \Gamma(\alpha+1)}:=\widetilde{R}
$$

and $W$ is bounded. Let $R>\max \{\widetilde{R}, r\}$; then, by Lemma 11, we have

$$
i\left(A, P_{R}, P\right)=1 \text {. }
$$

Now by the additivity of fixed point index, (41) and (48), we have

$$
i\left(A, P_{R} \backslash \overline{P_{r}}, P\right)=i\left(A, P_{R}, P\right)-i\left(A, P_{r}, P\right)=1 .
$$

Therefore $A$ has a fixed point in $P_{R} \backslash \overline{P_{r}}$, which is a positive solution of BVP (2).

The proof of Theorem 13 is completed.

For convenience, we set $M_{0}=\left(\int_{1 / 4}^{3 / 4} G(1, s) s^{\alpha-1} d s\right)^{-1}$.

Theorem 14. Assume that $f:[0,1] \times[0, \infty) \rightarrow[0, \infty)$ is continuous and satisfies the following conditions.

(F3) There exist $0<a<\lambda_{1}$ and $\delta>0$, such that

$$
f(t, x) \leq a x, \quad \forall t \in[0,1], 0 \leq x \leq \delta .
$$

(F4) There exist $b>M_{0}$ and $\delta<H \in \mathbb{R}^{+}$, such that

$$
f(t, x) \geq b x, \quad \forall t \in[0,1], x \geq H .
$$

Then, BVP (2) has at least one positive solution.

Proof. Let $T_{1} u=\lambda_{1} T u, u \in C[0,1]$, where $T$ is defined in (18). Then, $T_{1}: C[0,1] \rightarrow C[0,1]$ is a bounded linear operator; we have

$$
T_{1}(P) \subset P, \quad r\left(T_{1}\right)=1 .
$$

Choose $r \in(0, \delta)$, where $\delta$ is the constant in assumption (F3). For every $u \in \partial P_{r}$, from assumption (F3), we have

$$
\begin{aligned}
(A u)(t) & =\int_{0}^{1} G(t, s) f(s, u(s)) d s \\
& \leq a \int_{0}^{1} G(t, s) u(s) d s \\
& <\lambda_{1} \int_{0}^{1} G(t, s) u(s) d s \\
& =\lambda_{1}(T u)(t)=\left(T_{1} u\right)(t), \quad t \in[0,1] .
\end{aligned}
$$

Namely, $A u \leq T_{1} u, \forall u \in \partial P_{r}$.

Suppose that $A$ has no fixed point on $\partial P_{r}$ (otherwise, the proof is completed). Now we show that

$$
A u \neq \mu u, \quad \forall u \in \partial P_{r}, \mu \geq 1 .
$$

If it is not true, there exist $u_{1} \in \partial P_{r}$ and $\mu_{0}>1$ such that

$$
A u_{1}=\mu_{0} u_{1} \text {. }
$$

Then, $\mu_{0} u_{1}=A u_{1} \leq T_{1} u_{1}, \mu_{0}>1$. Therefore, we have

$$
\mu_{0}^{n} u_{1} \leq T_{1}^{n} u_{1} \leq\left\|T_{1}^{n}\right\|\left\|u_{1}\right\|, \quad(n=1,2, \ldots) .
$$

Thus, $\mu_{0}^{n} \leq\left\|T_{1}^{n}\right\|$. Using Gelfand's theorem, we have

$$
r\left(T_{1}\right)=\lim _{n \rightarrow \infty} \sqrt[n]{\left\|T_{1}^{n}\right\|} \geq \mu_{0}>1,
$$


which contradicts (52). Hence (54) holds; from Lemma 11, we have

$$
i\left(A, P_{r}, P\right)=1 \text {. }
$$

On the other hand, from (F4), we have

$$
f(t, x) \geq b x, \quad \forall t \in[0,1], x \geq H .
$$

We choose $R>\max \left\{4^{\alpha-1} H, r\right\}$. For every $u \in \partial P_{R}$, by the definitions of $P$ and $P_{R}$, we have $\|u\|=R$ and

$$
u(s) \geq s^{\alpha-1}\|u\| \geq\left(\frac{1}{4}\right)^{\alpha-1} R \geq H, \quad \forall s \in\left[\frac{1}{4}, \frac{3}{4}\right] .
$$

Hence, form (59), it follows that

$$
f(s, u(s)) \geq b u(s) \geq b s^{\alpha-1}\|u\|, \quad \forall s \in\left[\frac{1}{4}, \frac{3}{4}\right] .
$$

Now, by (11) and (61), we have

$$
\begin{aligned}
\|A u\| & \geq(A u)(1) \\
& =\int_{0}^{1} G(1, s) f(s, u(s)) d s \\
& \geq \int_{1 / 4}^{3 / 4} G(1, s) f(s, u(s)) d s \\
& \geq b\|u\| \int_{1 / 4}^{3 / 4} G(1, s) s^{\alpha-1} d s \\
& >M_{0}\|u\| \int_{1 / 4}^{3 / 4} G(1, s) s^{\alpha-1} d s=\|u\| .
\end{aligned}
$$

This means that

$$
\|A u\|>\|u\|, \quad \forall u \in \partial P_{R} .
$$

Hence, by Lemma 12, we have that

$$
i\left(A, P_{R}, P\right)=0 \text {. }
$$

Now by the additivity of fixed point index, (58) and (64), we have

$$
i\left(A, P_{R} \backslash \overline{P_{r}}, P\right)=i\left(A, P_{R}, P\right)-i\left(A, P_{r}, P\right)=-1 .
$$

Therefore $A$ has a fixed point in $P_{R} \backslash \overline{P_{r}}$, which is a positive solution of BVP (2).

The proof of Theorem 14 is completed.

Example 15. Consider the following fractional differential equations boundary value problem:

$$
\begin{gathered}
-D_{0^{+}}^{\alpha} u(t)=\frac{c u(t)}{\sqrt{1+u^{2}(t)}}, \quad t \in(0,1), \\
u(0)=u^{\prime}(0)=u^{\prime}(1)=0,
\end{gathered}
$$

where $2<\alpha \leq 3, c>0$ are constants. We assume that $c$ is large enough such that $c>\lambda_{1}$. Then it is easy to verify that

$$
f(t, x)=\frac{c x}{\sqrt{1+x^{2}}}, \quad t \in[0,1], x \geq 0
$$

satisfies conditions (F1) and (F2) of Theorem 13. Hence, by Theorem 13, (66) has at least one positive solution.
Example 16. Consider the fractional differential equations boundary value problem

$$
\begin{gathered}
-D_{0^{+}}^{\alpha} u(t)=d_{1} u(t)+d_{2} u^{2}(t), \quad t \in(0,1), \\
u(0)=u^{\prime}(0)=u^{\prime}(1)=0,
\end{gathered}
$$

where $2<\alpha \leq 3, d_{1}, d_{2}>0$ are constants. Let $0<d_{1}<\lambda_{1}$. We can easily see that

$$
f(t, x)=d_{1} x+d_{2} x^{2}, \quad t \in[0,1], x \geq 0
$$

satisfies conditions (F3) and (F4) of Theorem 14. By Theorem 14, (68) has at least one positive solution.

\section{Conflict of Interests}

The authors declare that there is no conflict of interests regarding the publication of this paper.

\section{Acknowledgments}

This research is supported by NNSF of China (11261053) and NSF of Gansu Province (1208RJZA129).

\section{References}

[1] S. G. Samko, A. A. Kilbas, and O. I. Marichev, Fractional Integral And Derivatives (Theory and Applications), Gordon and Breach, Yverdon, Switzerland, 1993.

[2] K. Miller and B. Ross, An Introduction to the Fractional Calculus and Fractional Differential Equations, Wiley, New York, NY, USA, 1993.

[3] I. Podlubny, Fractional Differential Equations, Mathematics in Science and Engineering, Academic Press, New York, NY, USA, 1999.

[4] Y. Wang, L. Liu, and Y. Wu, "Positive solutions for a nonlocal fractional differential equation," Nonlinear Analysis: Theory, Methods \& Applications, vol. 74, no. 11, pp. 3599-3605, 2011.

[5] C. S. Goodrich, "Existence and uniqueness of solutions to a fractional difference equation with nonlocal conditions," Computers \& Mathematics with Applications, vol. 61, no. 2, pp. 191-202, 2011.

[6] J. Mao, Z. Zhao, and N. Xu, "On existence and uniqueness of positive solutions for integral boundary value problems," Electronic Journal of Qualitative Theory of Differential Equations, vol. 16, pp. 1-8, 2010.

[7] D. Jiang and C. Yuan, "The positive properties of the Green function for Dirichlet-type boundary value problems of nonlinear fractional differential equations and its application," Nonlinear Analysis: Theory, Methods \& Applications, vol. 72, no. 2, pp. 710-719, 2010.

[8] S. Zhang and X. Su, "The existence of a solution for a fractional differential equation with nonlinear boundary conditions considered using upper and lower solutions in reverse order," Computers \& Mathematics with Applications, vol. 62, no. 3, pp. 1269-1274, 2011.

[9] B. Ahmad and J. J. Nieto, "Existence results for a coupled system of nonlinear fractional differential equations with threepoint boundary conditions," Computers \& Mathematics with Applications, vol. 58, no. 9, pp. 1838-1843, 2009. 
[10] Z. Bai and H. Lü, "Positive solutions for boundary value problem of nonlinear fractional differential equation," Journal of Mathematical Analysis and Applications, vol. 311, no. 2, pp. 495-505, 2005.

[11] S. Zhang, "Positive solutions for boundary-value problems of nonlinear fractional differential equations," Electronic Journal of Differential Equations, vol. 2006, no. 36, 12 pages, 2006.

[12] Z. Wei, Q. Li, and J. Che, "Initial value problems for fractional differential equations involving Riemann-Liouville sequential fractional derivative," Journal of Mathematical Analysis and Applications, vol. 367, no. 1, pp. 260-272, 2010.

[13] L. Lin, X. Liu, and H. Fang, "Method of upper and lower solutions for fractional differential equations," Electronic Journal of Differential Equations, vol. 100, pp. 1-13, 2012.

[14] C. Bai, "Impulsive periodic boundary value problems for fractional differential equation involving Riemann-Liouville sequential fractional derivative," Journal of Mathematical Analysis and Applications, vol. 384, no. 2, pp. 211-231, 2011.

[15] S. K. Ntouyas, G. Wang, and L. Zhang, "Positive solutions of arbitrary order nonlinear fractional differential equations with advanced arguments," Opuscula Mathematica, vol. 31, no. 3, pp. 433-442, 2011.

[16] W. C. Troy, "Solutions of third-order differential equations relevant to draining and coating flows," SIAM Journal on Mathematical Analysis, vol. 24, no. 1, pp. 155-171, 1993.

[17] Q. Yao and Y. Feng, "The existence of solution for a thirdorder two-point boundary value problem," Applied Mathematics Letters, vol. 15, no. 2, pp. 227-232, 2002.

[18] F. Bernis and L. A. Peletier, "Two problems from draining flows involving third-order ordinary differential equations," SIAM Journal on Mathematical Analysis, vol. 27, no. 2, pp. 515-527, 1996.

[19] A. A. Kilbas, H. M. Srivastava, and J. J. Trujillo, Theory and Applications of Fractional Differential Equations, Elsevier Science, Amsterdam, The Netherlands, 2006.

[20] M. El-Shahed, "Positive solutions for boundary value problem of nonlinear fractional differential equation," Abstract and Applied Analysis, vol. 2007, Article ID 10368, 8 pages, 2007.

[21] K. Deimling, Nonlinear Functional Analysis, Springer, Berlin , Germany, 1985.

[22] D. Guo and V. Lakshmikantham, Nonlinear Problems in Abstract Cones, Academic Press, New York, NY, USA, 1988. 


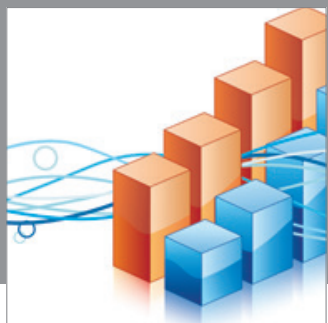

Advances in

Operations Research

mansans

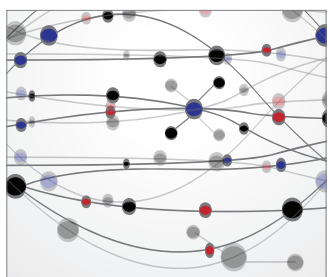

The Scientific World Journal
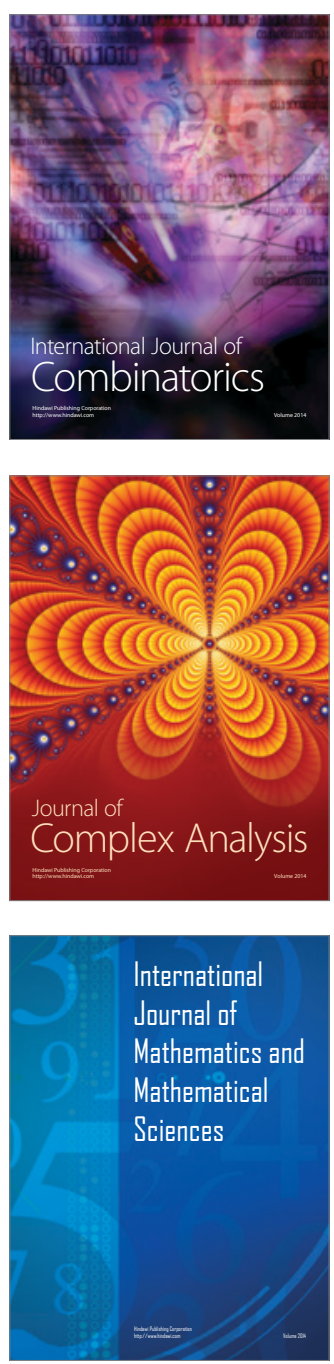
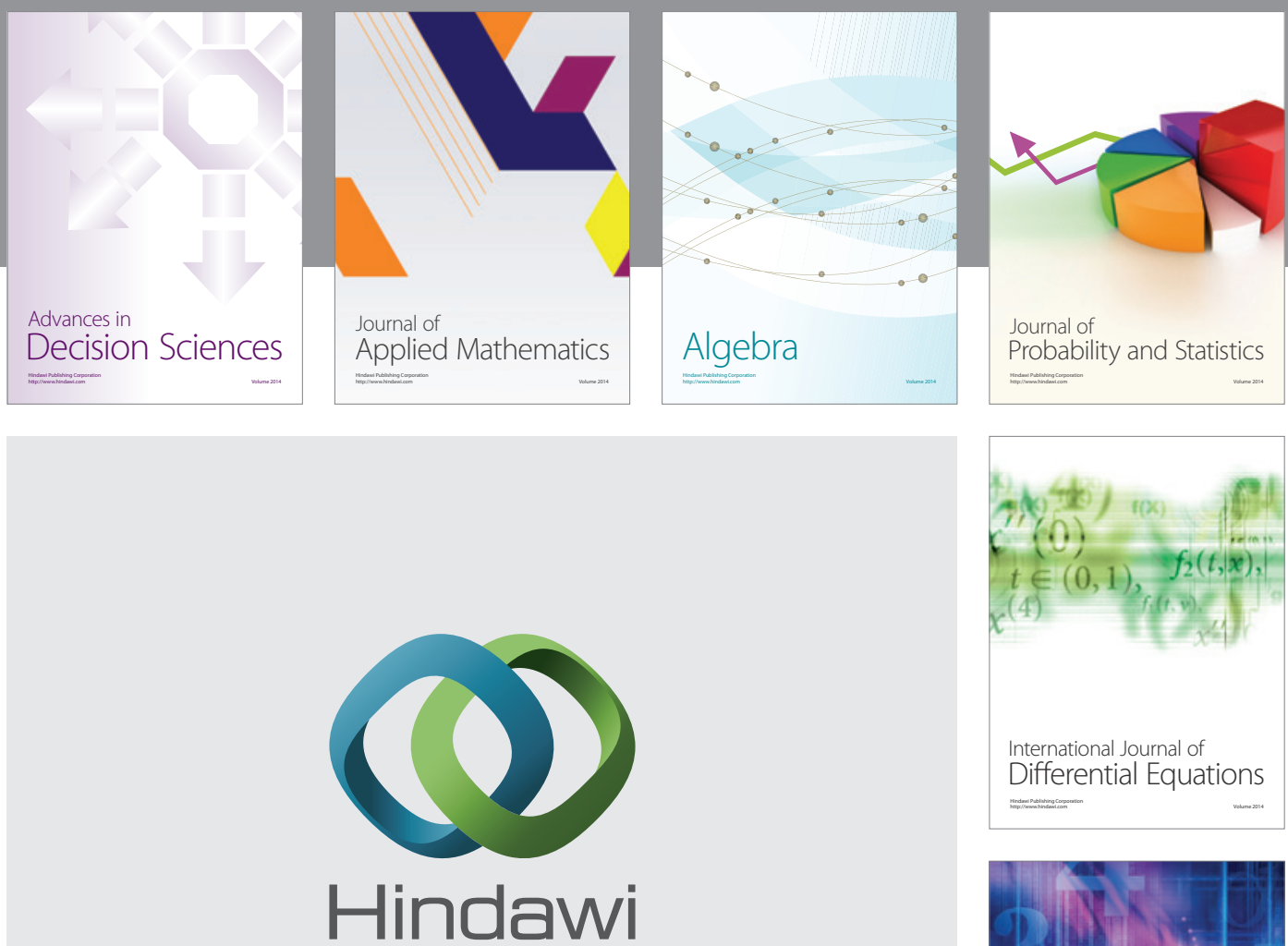

Submit your manuscripts at http://www.hindawi.com
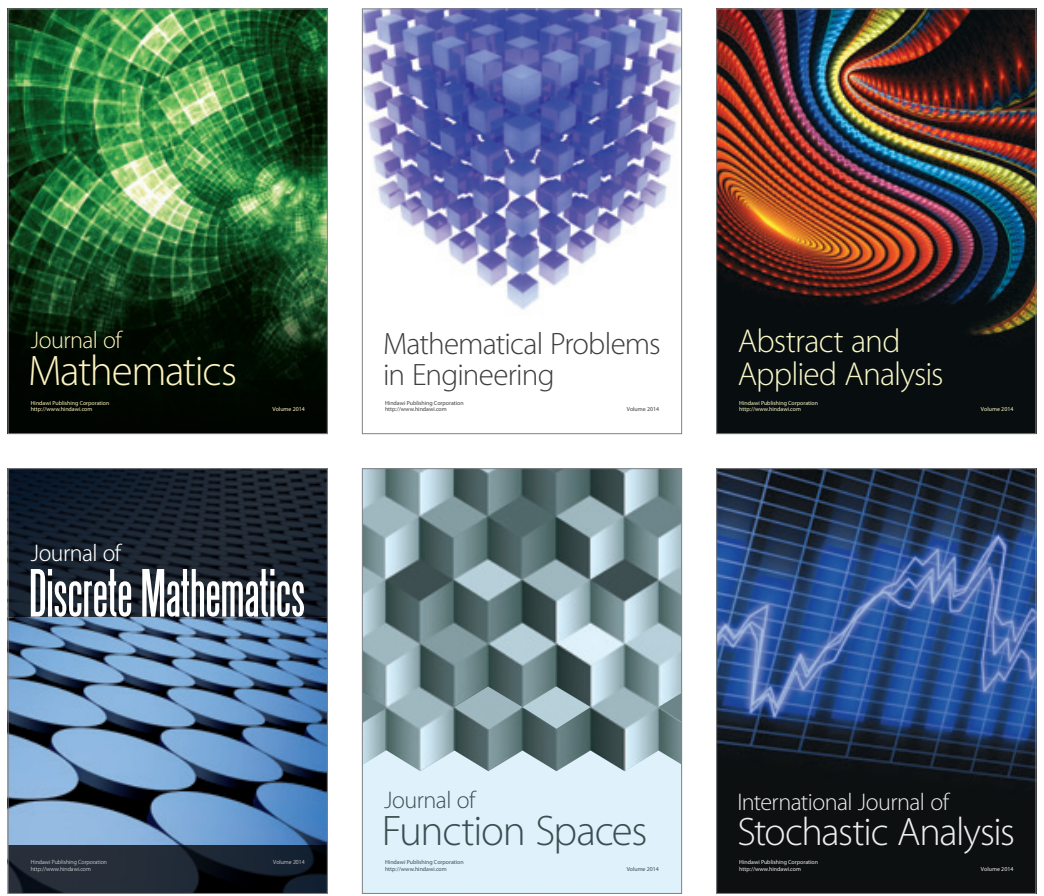

Journal of

Function Spaces

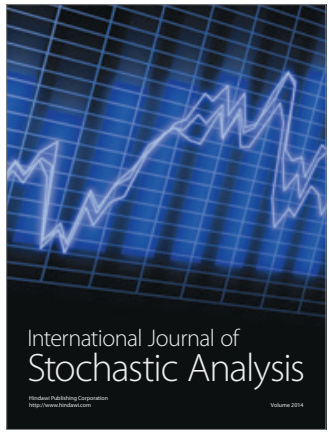

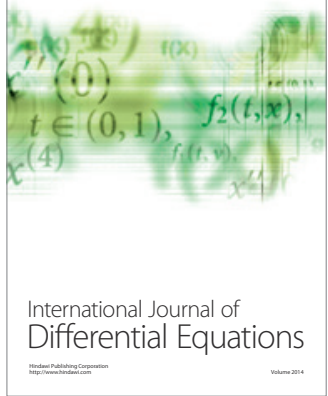
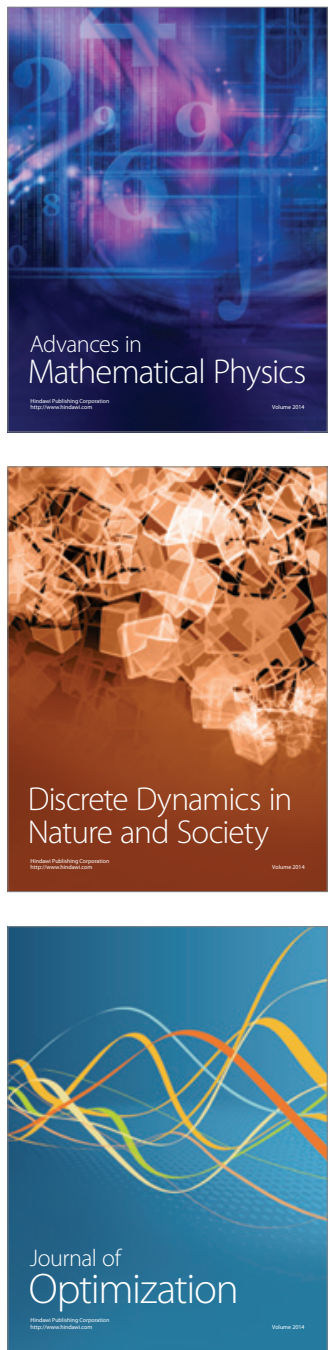